\title{
Differences in milk fat composition predicted by mid-infrared spectrometry among dairy cattle breeds in the Netherlands
}

\author{
M. H. T. Maurice-Van Eijndhoven,${ }^{*} \dagger^{1}$ H. Bovenhuis, $\dagger$ H. Soyeurt, $\neq \S$ and M. P. L. Calus ${ }^{*}$ \\ *Animal Breeding and Genomics Centre, Wageningen UR Livestock Research, PO Box 65, 8200 AB Lelystad, the Netherlands \\ †Animal Breeding and Genomics Centre, Wageningen University, PO Box 338, $6700 \mathrm{AH}$ Wageningen, the Netherlands \\ $\ddagger$ Animal Science Unit, Gembloux Agro-Bio Tech, University of Liège, 5030 Gembloux, Belgium \\ §National Fund for Scientific Research, 1000 Brussels, Belgium
}

\begin{abstract}
The aim of this study was to estimate breed differences in milk fatty acid (FA) profile among 5 dairy cattle breeds present in the Netherlands: Holstein-Friesian (HF), Meuse-Rhine-Yssel (MRY), Dutch Friesian (DF), Groningen White Headed (GWH), and Jersey (JER). For this purpose, total fat percentage and detailed FA contents in milk (14 individual FA and 14 groups of FA) predicted from mid-infrared spectra were used. Mid-infrared spectrometry profiles were collected during regular milk recording from a range of herds with different combinations of breeds, including both purebred and crossbred cows. The data set used for the analyses contained 41,404 records from a total of 24,445 cows. In total 7,626 cows were crossbreds belonging to the breeds HF, MRY, DF, GWH, and JER; 1,769 purebreds $(\geq 87.5 \%)$ belonging to the breeds MRY, DF, GWH, and JER; and the other 15,050 cows were HF. Breed effects were estimated using a single-trait animal model. The content in milk of short-chain FA C4:0, C6:0, C8:0, C10:0, C12:0, C14:0, and C16:0 was higher for JER and the content in milk of C16:0 was lower for GWH compared with the other breeds; when adjusting for breed differences in fat percentage, however, not all breed differences were significant. Breed differences were also found for cis-9 C14:1, cis-9 C16:1, C18:0, and a number of $\mathrm{C} 18$ unsaturated FA. In general, differences in fat composition in milk between HF, MRY, and DF were not significant. Jerseys tended to produce more saturated FA, whereas GWH tended to produce relatively less saturated FA. After adjusting for differences in fat percentage, breed differences in detailed fat composition disappeared or became smaller for several short- and medium-chain FA, whereas for several long-chain unsaturated FA, more significant breed differences were found. This indicates that short- and medium-chain FA are for all breeds more related to
\end{abstract}

Received June 1, 2012.

Accepted November 28, 2012.

${ }^{1}$ Corresponding author: myrthe.maurice-vaneijndhoven@wur.nl total fat percentage than long-chain FA. In conclusion, between breed differences were found in detailed FA composition and content of individual FA. Especially, for FA produced through de novo synthesis (short-chain FA, C12:0, C14:0, and partly C16:0) differences were found for JER and GWH, compared with the breeds HF, MRY, and DF.

Key words: milk, fatty acid, mid-infrared spectrometry, cattle breed

\section{INTRODUCTION}

Bovine milk fat is composed of a wide range of $\mathrm{FA}$, which can be distinguished based on their number of carbons, the saturation of their carbon chain, and the conformation of double bonds. These different FA can roughly be divided into SFA with no double bounds, which make up around $70 \%$ of the total milk fat, and unsaturated FA (UFA) with 1 (25\% MUFA) or multiple double bounds (5\% PUFA). The detailed FA composition in milk is variable and can differ between cows and herds (e.g., Stoop et al., 2008). Extending the knowledge on variation in detailed FA composition is of major interest for the dairy industry because of the expected effects of dairy fat intake on human health (Mensink et al., 2003; Palmquist et al., 2006) and associations between FA composition with milk processability (e.g., Smet et al., 2009) and individual methane emission (Dijkstra et al., 2011). The variation in FA composition in milk can be partly explained by differences in the diet of the cows (e.g., Baumgard et al., 2001; Sterk et al., 2011). Besides diet, a considerable part of the variation also has a genetic origin. For instance, Mele et al. (2009) reported heritabilities for individual FA in milk of Italian Holstein-Friesians (HF) ranging from 0.03 to 0.17 and Stoop et al. (2008) reported heritabilities for individual FA in milk of Dutch HF ranging from 0.22 to 0.71 . This indicates that a considerable part of the variation in FA composition is due to genetics. Breeding, therefore, can be a tool to change the FA composition in bovine milk. In addition to genetic variation within breeds, difference between dairy breeds in FA composi- 
tion might be relevant. Furthermore, identification of specific breed characteristics could provide arguments for breed conservation. Differences in FA composition between herds with different dairy breeds in the Netherlands were reported by Maurice-Van Eijndhoven et al. (2011). Breed differences in FA composition were also found by DePeters et al. (1995) in which differences were reported between HF, Jersey (JER), and Brown Swiss Beaulieu and Palmquist (1995), in which differences were reported between HF and JER, and Lawless et al. (1999) found differences for several individual FA between Irish HF, Dutch HF, Montbéliardes, and Normandes in Ireland. In the first study (Maurice-Van Eijndhoven et al., 2011), however, the structure of the data did not allow separation of breed and herd effects.

To accurately disentangle breed and herd effects, data across a range of herds with multiple combinations of breeds are needed. The latter is a major challenge if the majority of herds only have purebred cows from 1 breed. To be able to identify breed differences, a large number of records including detailed milk FA profiles are needed. Unfortunately, the most commonly used method to determine FA composition in milk is gas chromatography (GC). Gas chromatography is relatively expensive and time consuming and, therefore, less suitable to assess the detailed milk fat composition for large numbers of milk samples. An alternative method to predict FA composition is mid-infrared spectrometry (MIRS) as described by Soyeurt et al. (2007b), Rutten et al. (2009), and De Marchi et al. (2011). Mid-infrared spectrometry is less expensive and time consuming and commonly used by milk laboratories to analyze the major milk components such as fat and protein content, which makes MIRS attractive for routine prediction of FA and for large-scale experiments. For example, Soyeurt et al. (2007a) reported heritabilities calculated using individual FA predicted using MIRS profiles in milk of dairy cattle in the Walloon region of Belgium, ranging from 0.05 to 0.38. In another study of Soyeurt et al. (2006b), using predicted FA databased on MIRS, some breed differences in FA composition were reported among the dairy breeds dual-purpose Belgian Blue, HF, JER, Montbéliarde, and Meuse-Rhine-Yssel (MRY) participating in the Walloon milk recording in Belgium.

The aim of this paper is to identify breed differences in FA composition among the dairy cattle breeds HF, MRY, Dutch Friesian (DF), Groningen White Headed (GWH), and JER. This was achieved by comparing the predicted FA composition for those different cattle breeds in the Netherlands using a data set with MIRS profiles from regular milk recording, including a range of herds with different combinations of breeds, considering both purebred and crossbred animals.

\section{MATERIALS AND METHODS}

\section{Data Collection and Data Editing}

Mid-infrared spectrometry profiles of milk samples were collected via the Dutch milk recording system of CRV BV (Arnhem, the Netherlands) between October and December 2006. Samples were treated immediately with $0.03 \%$ (wt/wt) sodium azide to avoid microbiological growth. The MIRS profiles were obtained using 3 Fourier-transformed interferogram machines (MilkoScan FT 6000; Foss Electric A/S, Hillerød, Denmark) at the laboratory of Qlip N.V. (Leusden, the Netherlands). The sampled herds were a random representation of all herds participating in the milk recording system of CRV BV.

The initial data set contained 372,429 test-day records of 230,995 cows. Data-editing steps included the deletion of records and cows for the following reasons: less than $75 \%$ of the breed composition known, unknown sire, incomplete milk recording data (e.g., unknown birthdate or DIM), 2 records from the same cow on the same sample date, cows with records in more than 1 herd, cows reported sick at sampling date, cows in parity 11 or higher, cows before 5 or after 365 d in lactation, and cows in herds with less than 5 purebred cows of the same breed (HF, MRY, DF, or GWH) per herd. To detect records with possible errors, due to, for example, swapped samples, fat content recorded via the regular milk control (predicted by QLIP N.V.) was compared with the values obtained using the RobustMilk prediction equations (Soyeurt et al., 2011). The correlation coefficient between fat content predicted by QLIP N.V. and fat content predicted using the RobustMilk prediction equations was 0.996 . When the absolute difference in both predictions for fat percentage was more than 0.35 the record was removed. Finally, complete records with extreme outliers in at least 1 of all predicted traits $( \pm 5 \mathrm{SD}$ of the mean) were deleted. After these editing steps, the data set contained 307,656 records.

A large number of these records were from $\mathrm{HF}$ animals from herds without crossbreds or animals from breeds other than HF. Because these records do not contribute to the breed estimates and makes the data set heavily unbalanced, only animals from herds with at least 3 animals with $>25 \%$ genes from MRY, DF, GWH, or JER were kept in the data set. The final data set used for the analyses contained 41,404 records of 24,445 cows from 445 farms. A total of 7,626 cows were crossbreds belonging to the breeds HF, MRY, DF, GWH, and JER; 1,769 purebreds $(\geq 87.5 \%)$ belonging to the breeds MRY, DF, GWH, and JER; and the other 15,050 cows were HF. 


\section{Predicting FA Composition}

The MIRS profiles were used to predict the total fat percentage and detailed milk fat composition of 14 individual FA (C4:0, C6:0, C8:0, C10:0, C12:0, C14:0, cis-9 C14:1, C16:0, cis-9 C16:1, C18:0, cis-9 C18:1, cis-9,12 C18:2, cis-9,12,15 C18:3, and cis-9,trans-11 C18:2) and the 14 groups of FA [total trans C18:1, total cis C18:1, total C18:2, total trans C18, total SFA, total MUFA, total PUFA, total UFA, short-chain FA (SCFA), medium-chain FA (MCFA), long-chain FA (LCFA), total n-3 FA, total n-6 FA, and total branched-chain FA (BCFA)]. The calibration equations used to predict these FA were updated versions of the calibration equations of Soyeurt et al. (2011) based on 1,236 milk samples. The method used to relate MIRS spectra to FA data was partial least square regression after a firstderivative pretreatment on spectral data to correct the baseline drift. An external validation of the calibration equations used to predict FA in this study was published previously (Maurice-Van Eijndhoven et al., 2013). Results from that validation study indicate that the FA C4:0 to C16:0 and the groups SFA, SCFA, and MCFA can be predicted with high accuracy $\left(\mathrm{R}^{2}>0.80\right)$; cis-9 C14:1, cis-9 C16:1, and C18:0 can be predicted with moderate accuracy $\left(\mathrm{R}^{2}=0.48-0.80\right)$; and $\mathrm{C} 17: 0$ can be predicted with lower accuracy $\left(\mathrm{R}^{2}=0.15-0.73\right)$. Only FA were evaluated when the coefficient of determination was at least 0.60 over all breeds in this external validation, and for those not validated in that study, the cross-validation coefficient of determination of the calibration equations used was required to be at least 0.70 [the approach to calculate the cross-validation coefficient of determination was described by Soyeurt et al. (2011)]. The definition of the groups of FA and the predictability of the calibration equations by means of the (cross-validation) coefficient of determination are given in Table 1.

\section{Statistical Analysis}

Breed effects were estimated in ASReml 3.0 (Gilmour et al., 2009) using the following animal models:

$$
\begin{aligned}
& y_{i j k l m n o p q r s t u}=\mu+b_{1} \times \mathrm{DIM}_{i}+b_{2} \times \exp ^{-0.05 \times \mathrm{DIM}_{i}}+\text { parity }_{j}+b_{3} \\
& \times \operatorname{age}_{k}\left(\text { parity }_{j}\right)+h t d_{l}+b_{4} \times H F_{m}+b_{5} \times M R Y_{n}+b_{6} \times D F_{o}+b_{7} \\
& \times G W H_{p}+b_{8} \times J E R_{q}+b_{9} \times H E T_{r}+b_{10} \times R E C_{s}+\text { cow }_{t} \\
& +e_{i j k l m n o p q r s t}
\end{aligned}
$$

in this study referred to as the FAT - model, and

$$
\begin{aligned}
& y_{i j k l m n o p q r s t u v}=\mu+b_{1} \times \mathrm{DIM}_{i}+b_{2} \times \exp ^{-0.05 \times \mathrm{DIM}_{i}}+\text { parity }_{j}+b_{3} \\
& \times \operatorname{age}_{k}\left(\text { parity }_{j}\right)+h t d_{l}+b_{4} \times H F_{m}+b_{5} \times M R Y_{n}+b_{6} \times D F_{o}+b_{7} \\
& \times G W H_{p}+b_{8} \times J E R_{q}+b_{9} \times H E T_{r}+b_{10} \times R E C_{s}+b_{11} \\
& \times \operatorname{fatperc}_{t}\left(H F_{m}\right)+b_{12} \times \operatorname{fatperc}_{t}\left(M R Y_{n}\right)+b_{13} \\
& \times \operatorname{fatperc}_{t}\left(D F_{o}\right)+b_{14} \times \operatorname{fatperc}_{t}\left(G W H_{p}\right)+b_{15} \\
& \times \operatorname{fatperc}_{t}\left(J E R_{q}\right)+\operatorname{cow}_{u}+e_{i j k l m n o p q r s t u}
\end{aligned}
$$

in this study referred to as the FAT+ model, where $y_{i j k l m n o p q r s t u}(v)$ was the dependent variable for cow $t(u)$ in DIM $i$, with parity $j$, calving age $k$, producing at herd test date $l$, and having a breed composition mnopq; $\mu$ was the overall mean of the model; $b_{1}$ was the fixed regression coefficient on $\mathrm{DIM}_{i}$ and $b_{2}$ was the fixed regression coefficient on $\mathrm{DIM}_{i}$ modeled with a Wilmink

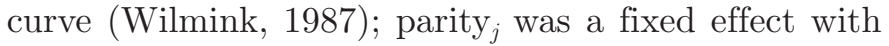
4 classes for corresponding lactation numbers of parity 1,2 , and 3 and the fourth class including parities 4 to $10 ; b_{3}$ was the fixed regression coefficient on age ${ }_{k}$, which was calving age in days, within the $j$ th parity; $h t d_{l}$ was a fixed effect defining 841 groups of cows sampled in the same herd on the same sample date; $b_{4}, b_{5}, b_{6}, b_{7}$, and $b_{8}$ were the fixed regression coefficients on, respectively, $H F_{m}, M R Y_{n}, D F_{o}, G W H_{p}$, and $J E R_{q}$, which were the estimated percentages of genes belonging to each of those breeds; $b_{9}$ was the fixed regression on $H E T_{r}$, which was the estimated percentage of heterosis; $b_{10}$ was the fixed regression on $R E C_{s}$, which was the estimated percentage of recombination loss effect; $b_{11}$ to $b_{15}$ were the fixed regression coefficients of fatperc $c_{t}$, which was the total fat percentage in milk, within the breeds HF, MRY, DF, GWH, and JER; $\operatorname{cow}_{t(u)}$ was a random permanent environmental effect (no genetic relationships included) of cow $t(u)$; and $e_{i j k l m n o p q r s t}(u)$ was the random residual effect. Heterosis was calculated as function of the degree of heterozygosity of animals and $R E C$ was derived from the heterozygosity of parental gametes, whose calculations are both described by Van Der Werf and De Boer (1989).

For each trait model, assumptions were checked for equal variances, independency of the phenotypic values, and normality of the residuals. Only for the trait cis-9 C18:1 did the residuals substantially deviate from normality. To avoid problems in the comparison of results due to differences in scales resulting from, for example, log transforming the values, and transforming results back to the original scale, this trait was simply analyzed on the original scale. Reporting cis-9 C18:1 on the original scale is in line with others who analyzed 
Table 1. Definition of the groups of FA and the validation coefficient of determination of the prediction

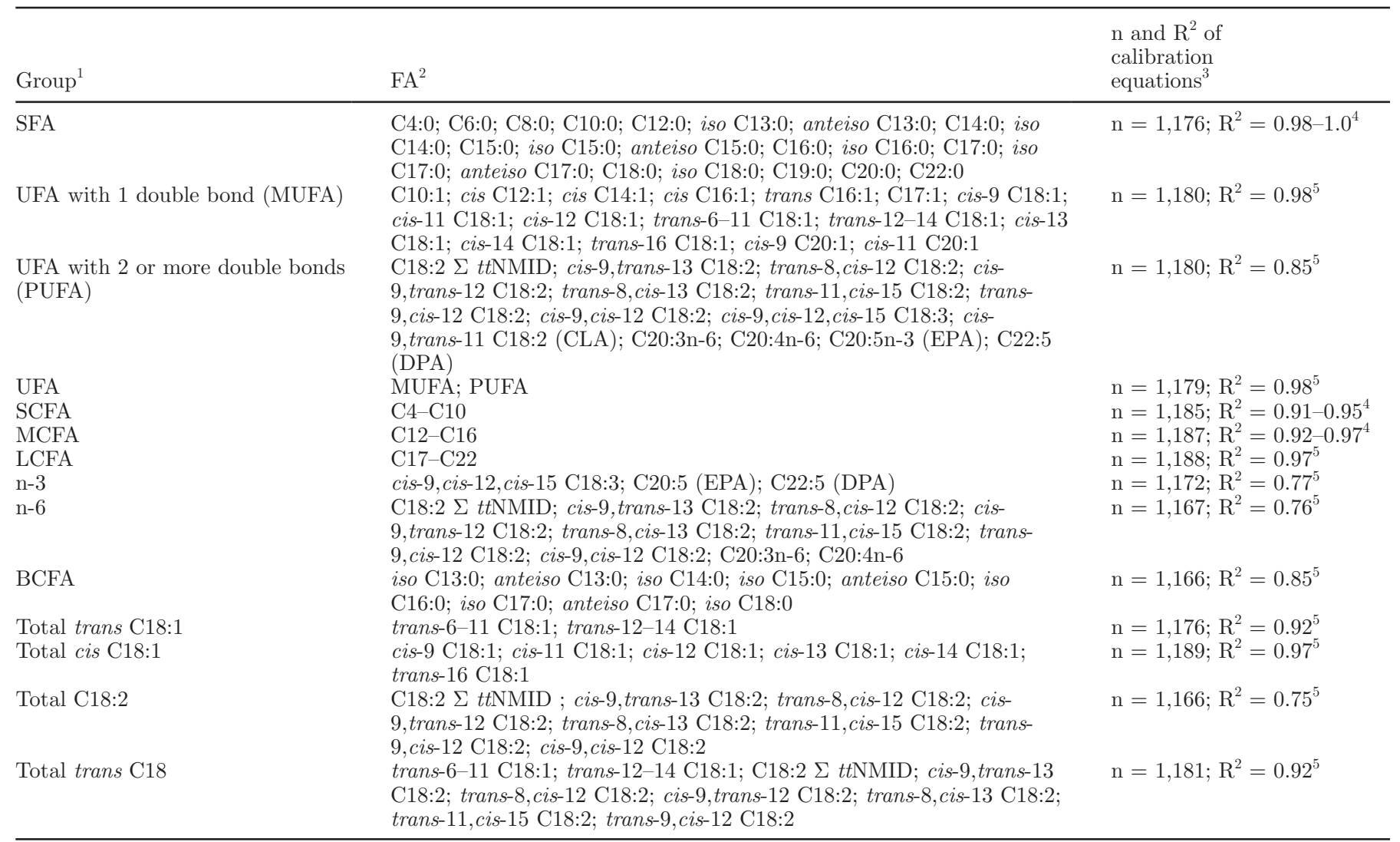

${ }^{1} \mathrm{UFA}=$ unsaturated FA; SCFA = short-chain FA; MCFA = medium-chain FA; LCFA = long-chain FA; BCFA = branched-chain FA.

${ }^{2} \mathrm{CLA}=$ conjugated linoleic acid; EPA = eicosapentaenoic acid; DPA = docosapentaenoic acid; $t$ NMID = trans, trans non-methylene-interrupted diene.

${ }^{3} \mathrm{n}=$ the number of samples included in the calibration equation; $\mathrm{R}^{2}=$ the cross-validation coefficient of determination of the calibration equation.

${ }^{4}$ Range of validation $\mathrm{R}^{2}$ of the calibration equations for the group of FA based on separate validation data sets of the different breeds Groningen White Headed, Dutch Friesian, Meuse-Rhine-Yssel, and Jersey (Maurice-Van Eijndhoven et al., 2013).

${ }^{5}$ The cross-validation $\mathrm{R}^{2}$ of the calibration equations. The cross-validation approach to calculate the $\mathrm{R}^{2}$ is described by Soyeurt et al. (2011).

the same trait (e.g., Stoop et al., 2008; Rutten et al., 2009).

Breed effects were estimated by calculating the predicted means of all traits for each breed in the third parity and $156 \mathrm{~d}$ in lactation. For the FAT+ model, means were predicted at the average fat percentage of 4.70. To test if the observed differences in FA content between breeds were significant a Student's $t$-test was performed. For the calculation of the $t$-test statistic, the standard error of difference was obtained from ASReml 3.0 software (Gilmour et al., 2009). The $P$-values were obtained using a 2-tailed Student's $t$-distribution with $n$ degrees of freedom, where $n$ was conservatively chosen to be the number of sires of the smallest breed of the 2 breeds that were compared $(\mathrm{n}=596 \mathrm{MRY} ; 156$ FH; 66 G; and 80 JER sires).

The data included crossbred animals; therefore, heterosis and recombination effects were included in the model. To test whether these effects were significantly different from zero, a Student's $t$-test was performed using the same method as described above to test the breed differences and with 24,445 (total number of cows) degrees of freedom.

\section{RESULTS}

\section{Production Traits}

The unadjusted production data shows that HF produced the highest daily milk yield, followed by JER, DF, MRY, and GWH (Table 2). When assessing the results based on the FAT-, model only the milk yield of HF differed significantly $(P<0.05)$ from milk yields of MRY, GWH, and JER (Table 3). The results of the FAT - model show that the total fat percentage in milk for JER was significantly higher and for GWH sig- 
Table 2. The number of cows and herds, and means \pm SD of parity, calving age, DIM, production traits, individual FA, and groups of FA for the crossbred and purebred cows $(\geq 50 \%)$ in the unadjusted data

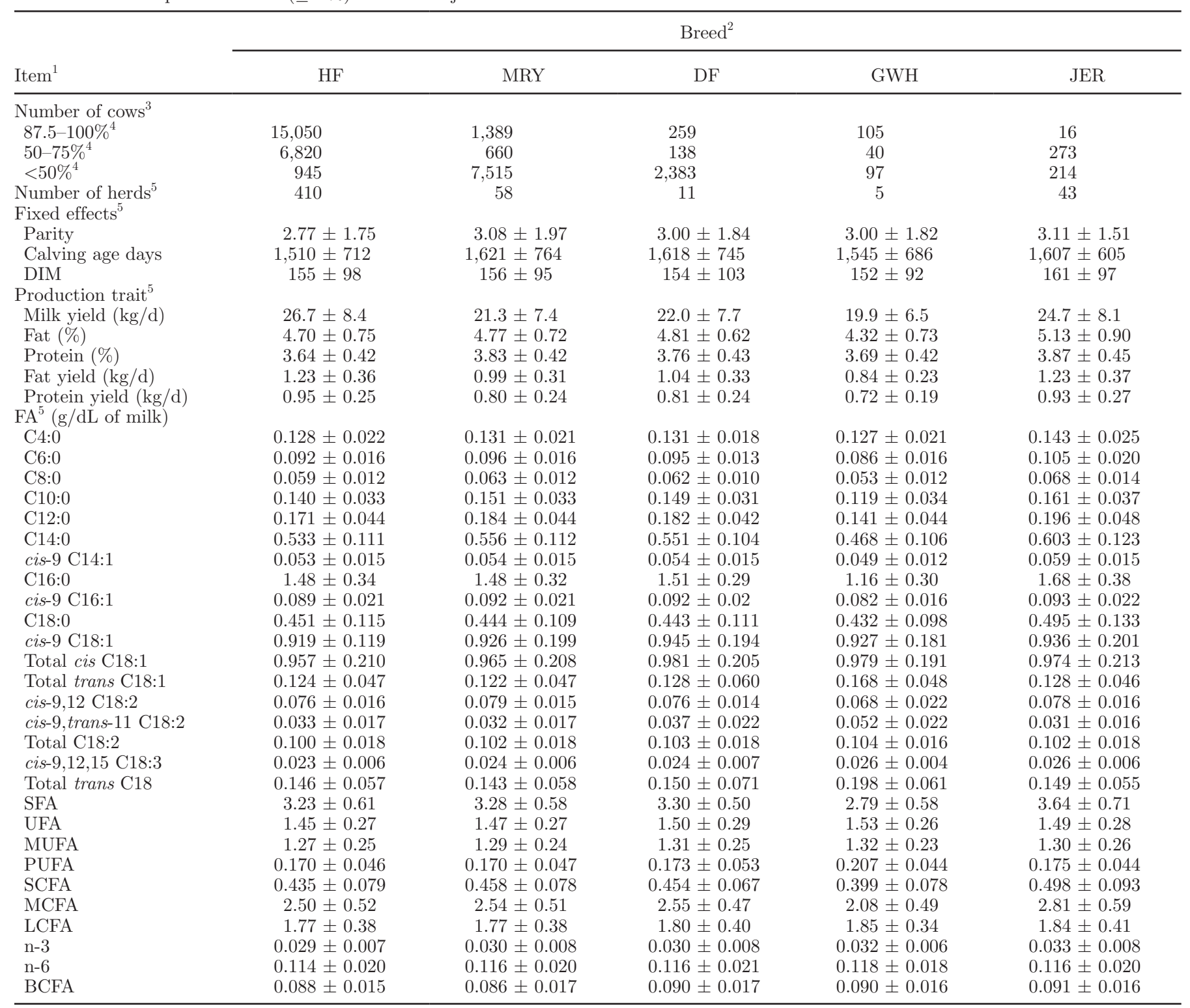

${ }^{1} \mathrm{UFA}=$ unsaturated FA; SCFA = short-chain FA; MCFA = medium-chain FA; LCFA = long-chain FA; BCFA = branched-chain FA.

${ }^{2} \mathrm{HF}=$ Holstein-Friesian; MRY = Meuse-Rhine-Yssel; DF = Dutch Friesian; GWH = Groningen White Headed; JER = Jersey.

${ }^{3}$ Note that crossbred animals are counted twice (i.e., an animal that is $50 \%$ HF and $50 \%$ MRY appears in the row $50-75 \%$ for both HF and MRY).

${ }^{4}$ Recorded breed percentages are expressed as multiples of $1 / 8$.

${ }^{5}$ Values include animals with $\geq 50 \%$ genes from HF, MRY, DF, GWH, or JER.

nificantly lower compared with the other breeds, which was in line with differences observed in the unadjusted data. Jerseys also produced a significant higher protein percentage.

\section{Individual FA C4:0 to C18:0}

The predicted means for $\mathrm{C} 4: 0$ to $\mathrm{C} 18: 0$ are shown in Table 3. When assessing the results based on the FAT- model, JER produced significantly higher contents for all of these individual FA than the other breeds. Using the FAT+ model, thus adjusting for differences in fat content, the predicted means of cis-9 C16:1 for JER were significantly lower compared with those for HF, MRY, and DF and tended to be lower compared with GWH $(P=0.08)$, and the predicted means of $\mathrm{C} 18: 0$ for JER tended to be lower compared with those for HF, MRY, and GWH. The predicted means of GWH for 
Table 3. Predicted means of 5 production traits and 10 individual FA for each breed ${ }^{1}$

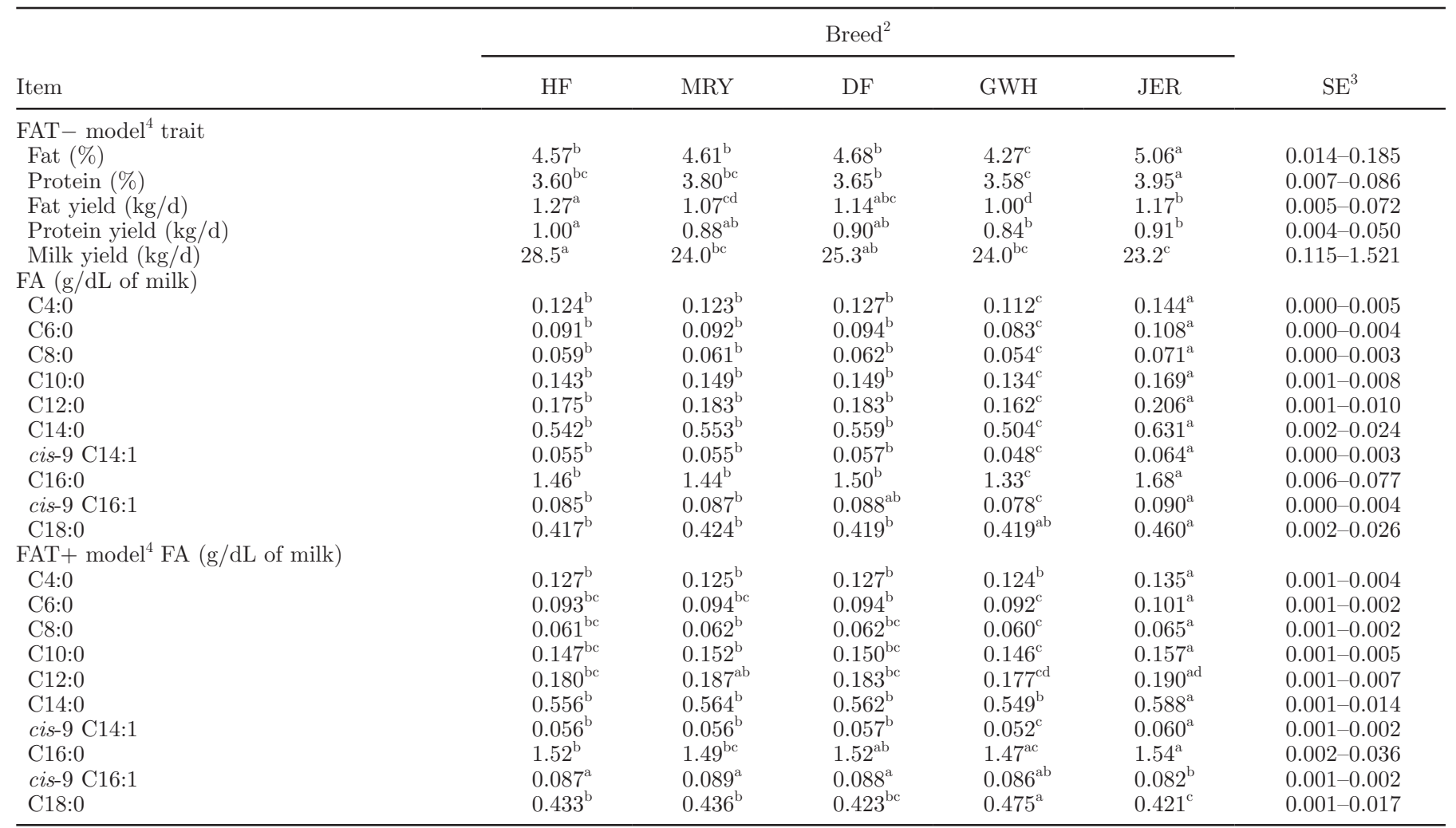

${ }^{\mathrm{a}-\mathrm{d}}$ Values with different superscripts within a row indicate significant differences of predicted means at $P<0.05$.

${ }^{1}$ Predicted means of all traits for each breed at the third parity and at 156 DIM in both models, and for the FAT + model, traits were predicted at a fat percentage of 4.70 .

${ }^{2} \mathrm{HF}=$ Holstein-Friesian; MRY $=$ Meuse-Rhine-Yssel; DF $=$ Dutch Friesian; GWH = Groningen White Headed; JER $=$ Jersey.

${ }^{3}$ Range of SE of the predicted means.

${ }^{4}$ Using the FAT - model, means were predicted regardless of the level of total fat percentage, and using the FAT + model, means were predicted including a fixed regression coefficient of the total fat percentage in milk within the breeds HF, MRY, DF, GWH, and JER.

C4:0 to C16:0 and cis-9 C16:1 were significantly lower $(P<0.05)$ when assessing the results of the FATmodel compared with all other breeds. When assessing the results of the FAT+ model, GWH also showed the lowest predicted means for C4:0 to C16:0, although not all comparisons with the other breeds were significant. The GWH breed produced the highest content of C18:0 when adjusting for differences in fat content.

\section{C18 UFA}

The predicted means of the individual C18 UFA and groups of these FA are in Table 4. Assessing the predicted means, whether adjusting for differences in fat percentage $(\mathrm{FAT}+)$ or not (FAT-), JER showed a significantly higher predicted mean for cis-9,12,15 C18:3. Jerseys produced a higher content of trans C18 FA compared with HF and MRY; however, this effect disappeared when adjusting for fat percentage in the model. Evaluating the results of the FAT+ model, GWH generally showed the highest predicted means for the C18 UFA; however, these predicted means were only in several comparisons significantly different from those of the other breeds.

\section{Groups of FA}

The predicted means of the groups of FA are shown in Table 5. Assessing the results of the FAT- model, JER showed significantly higher predicted means for all traits except for the group of MUFA and n- 6 FA. When adjusting for differences in fat percentage (the FAT+ model), predicted means of SFA, SCFA, and MCFA for JER were significantly higher compared with all other breeds and predicted means of PUFA (for JER) were significantly higher compared with HF and MRY. For the FAT + model, predicted means of UFA, MUFA, LCFA, and $\mathrm{n}-6$ for JER were significantly lower than those for the other breeds. The predicted means of all groups of FA for GWH, except for n-3 and LCFA, were significantly lower compared with the other breeds using the FATmodel, whereas for the FAT+ model, this was only still 
Table 4. Predicted means of 4 individual C18 unsaturated FA and 4 groups of C18 unsaturated FA for each breed ${ }^{1}$

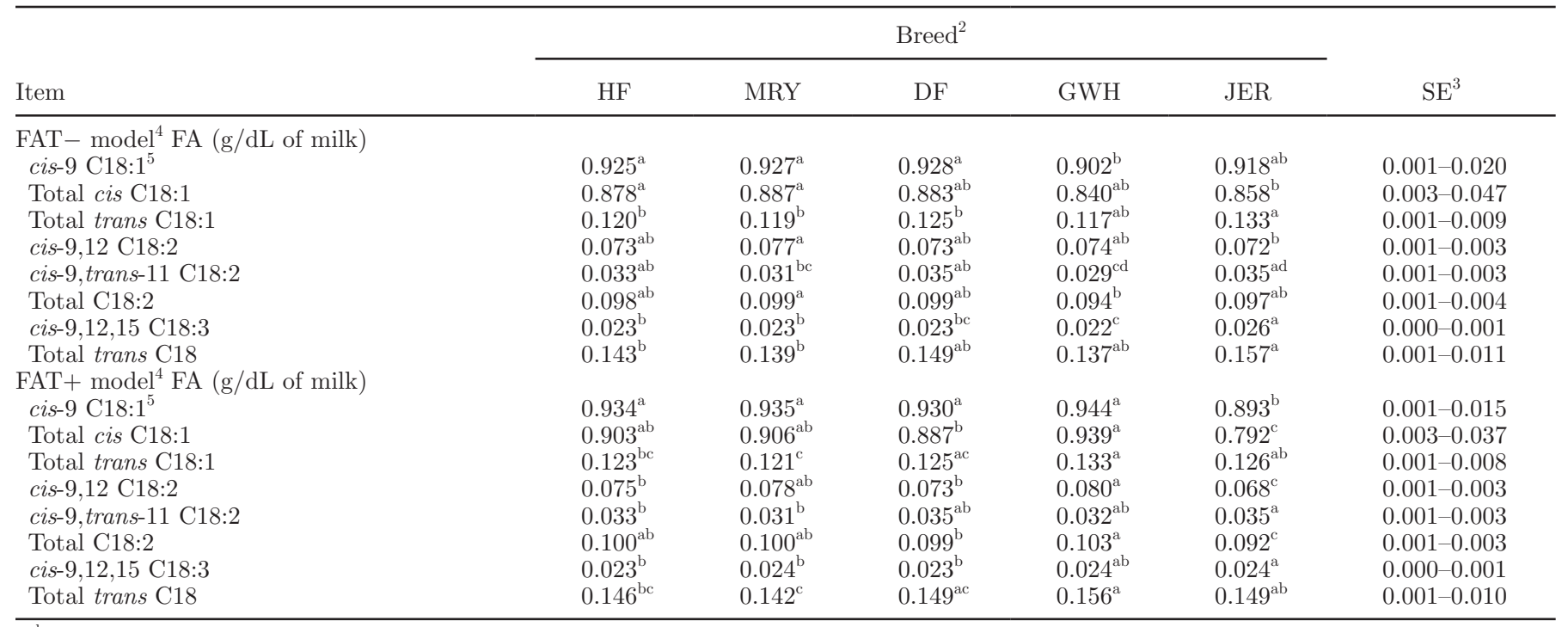

\footnotetext{
$\overline{\mathrm{a}-\mathrm{d}}$ Values with different superscripts within a row indicate significant differences of predicted means at $P<0.05$.

${ }^{1}$ Predicted means of all traits for each breed at the third parity and at 156 DIM in both models, and for the FAT + model, traits were predicted at a fat percentage of 4.70 .

${ }^{2} \mathrm{HF}=$ Holstein-Friesian; MRY = Meuse-Rhine-Yssel; DF = Dutch Friesian; GWH = Groningen White Headed; JER = Jersey.

${ }^{3}$ Range of SE of the predicted means.

${ }^{4}$ Using the FAT - model, means were predicted regardless of the level of total fat percentage, and using the FAT + model, means were predicted including a fixed regression coefficient of the total fat percentage in milk within the breeds HF, MRY, DF, GWH, and JER.

${ }^{5}$ To test the possible breed differences, the dependent variable was transformed using the natural logarithm. The predicted means given are transformed back to the original scale and the SE are on the scale of the natural logarithm.
}

the case for SCFA compared with MRY, DF, and JER and for MCFA compared with HF, DF, and JER.

\section{Heterosis and Recombination}

The estimates of the heterosis and recombination effects are shown in Table 6 . Heterosis was shown to be significant $(P<0.05)$ in both FAT - and FAT + models for the individual FA C4:0 to C10:0 and cis-9,trans-11 C18:2, and the group of FA SCFA. The recombination effect within the FAT - model was highly significant for most FA and groups of FA, except for the conjugated linoleic acid cis-9,trans-11 C18:2, total trans C18:1, and total trans $\mathrm{C} 18$. Within the model, adjusting for the differences in fat content $(\mathrm{FAT}+)$, the recombination effect was significant for 15 of the traits including C4:0 to C14:0. For almost all traits, both heterosis and recombination had positive effects when estimated with the FAT - model. When using the FAT+ model, heterosis showed slightly more positive than negative effects, and recombination had negative effects in most cases, and was in fact negative in all situations where it had a significant effect.

\section{DISCUSSION}

In this discussion section, first the use of MIRS profiles and the data structure is discussed. Thereafter, our results for different groups of FA are discussed and compared with published results. In published studies, either FA were evaluated as proportion of the total fat $(\mathrm{g} / 100 \mathrm{~g}$ of total fat), or as milk content (g/dL of milk). In our study, results are published as content in milk. Although predicted means of studies in which FA are evaluated as proportion of the total fat cannot directly be compared with our results, the direction of breed differences in other studies evaluating proportions in fat can be compared with the direction of the breed differences of the FAT+ model in our study. Comparisons to published results are performed for 3 groups of FA. The first group comprises FA that are formed through de novo synthesis (all SCFA and from all MCFA only $\mathrm{C} 12: 0$ and $\mathrm{C} 14: 0$ ), the second comprises the C16:0 FA that arises in milk through de novo synthesis and uptake from blood circulation, and the third group comprises FA that arise in milk by uptake from blood circulation (FA longer than 16 carbons in length). 
Table 5. Predicted means of 10 groups of FA for each breed ${ }^{1}$

\begin{tabular}{|c|c|c|c|c|c|c|}
\hline Item $^{2}$ & \multicolumn{5}{|c|}{ Breed $^{3}$} & $\mathrm{SE}^{4}$ \\
\hline \multicolumn{7}{|c|}{ FAT - model $^{5}$ FA ( $\mathrm{g} / \mathrm{dL}$ of milk $)$} \\
\hline UFA & $1.37^{\mathrm{a}}$ & $1.38^{\mathrm{a}}$ & $1.38^{\mathrm{a}}$ & $1.30^{\mathrm{b}}$ & $1.37^{\mathrm{a}}$ & $0.004-0.062$ \\
\hline MUFA & $1.19^{\mathrm{a}}$ & $1.20^{\mathrm{a}}$ & $1.21^{\mathrm{a}}$ & $1.12^{\mathrm{b}}$ & $1.19^{\mathrm{a}}$ & $0.004-0.056$ \\
\hline PUFA & $0.168^{\mathrm{b}}$ & $0.168^{\mathrm{b}}$ & $0.172^{\mathrm{b}}$ & $0.159^{\mathrm{c}}$ & $0.184^{\mathrm{a}}$ & $0.001-0.009$ \\
\hline $\mathrm{n}-3$ & $0.029^{\mathrm{b}}$ & $0.030^{\mathrm{b}}$ & $0.029^{\mathrm{b}}$ & $0.028^{\mathrm{b}}$ & $0.033^{\mathrm{a}}$ & $0.000-0.002$ \\
\hline$n-6$ & $0.111^{\mathrm{a}}$ & $0.112^{\mathrm{a}}$ & $0.112^{\mathrm{a}}$ & $0.106^{\mathrm{b}}$ & $0.111^{\mathrm{a}}$ & $0.000-0.004$ \\
\hline $\mathrm{BCFA}$ & $0.089^{b}$ & $0.086^{\mathrm{b}}$ & $0.091^{\mathrm{ab}}$ & $0.081^{\mathrm{c}}$ & $0.093^{\mathrm{a}}$ & $0.000-0.003$ \\
\hline \multicolumn{7}{|c|}{ FAT + model $^{5}$ FA (g/dL of milk) } \\
\hline SFA & $3.29^{\mathrm{b}}$ & $3.29^{\mathrm{b}}$ & $3.30^{\mathrm{b}}$ & $3.28^{\mathrm{b}}$ & $3.40^{\mathrm{a}}$ & $0.003-0.046$ \\
\hline UFA & $1.40^{\mathrm{a}}$ & $1.40^{\mathrm{a}}$ & $1.39^{\mathrm{a}}$ & $1.44^{\mathrm{a}}$ & $1.28^{\mathrm{b}}$ & $0.003-0.043$ \\
\hline $\mathrm{n}-3$ & $0.030^{\mathrm{c}}$ & $0.030^{\mathrm{bc}}$ & $0.030^{\mathrm{c}}$ & $0.032^{\mathrm{a}}$ & $0.031^{\mathrm{ab}}$ & $0.000-0.001$ \\
\hline $\mathrm{n}-6$ & $0.114^{\mathrm{a}}$ & $0.114^{\mathrm{a}}$ & $0.112^{\mathrm{a}}$ & $0.116^{\mathrm{a}}$ & $0.105^{\mathrm{b}}$ & $0.001-0.003$ \\
\hline BCFA & $0.091^{\mathrm{a}}$ & $0.087^{\mathrm{a}}$ & $0.092^{\mathrm{a}}$ & $0.089^{\mathrm{ab}}$ & $0.089^{\mathrm{b}}$ & $0.001-0.002$ \\
\hline
\end{tabular}

\footnotetext{
${ }^{\mathrm{a}-\mathrm{c}}$ Values with different superscripts within a row indicate significant differences of predicted means at $P<0.05$.

${ }^{1}$ Predicted means of all traits for each breed at the third parity and at 156 DIM in both models, and the FAT + model traits were predicted at a fat percentage of 4.70 .

${ }^{2} \mathrm{UFA}=$ unsaturated FA; SCFA = short-chain FA; MCFA = medium-chain FA; LCFA = long-chain FA; BCFA = branched-chain FA.

${ }^{3} \mathrm{HF}=$ Holstein-Friesian; MRY = Meuse-Rhine-Yssel; DF = Dutch Friesian; GWH = Groningen White Headed; JER = Jersey.

${ }^{4}$ Range of standard errors of the predicted means.

${ }^{5}$ Using the FAT - model, means were predicted regardless of the level of total fat percentage, and using the FAT+ model, means were predicted including a fixed regression coefficient of the total fat percentage in milk within the breeds HF, MRY, DF, GWH, and JER.
}

\section{MIRS Profiles}

The detailed milk fat composition was predicted based on MIRS profiles. The MIRS profiles were directly obtained from milk. In our study, results are, therefore, given on milk basis ( $\mathrm{g}$ of fat/dL of milk). Recalculation to fat basis leads to a considerable decrease in the accuracy, presumably due to an accumulation of bias. The decrease in accuracy when predicting results on fat basis from MIRS profiles was shown by Soyeurt et al. (2006a) and Rutten et al. (2009). The predictive ability of the calibration equations in milk of MRY, DF, GWH, and JER in the Netherlands has been investigated previously using an independent data set (Maurice-Van Eijndhoven et al., 2013). In that study, the majority of FA and groups of FA were predicted with moderate to high accuracy (based on the coefficient of determination $>0.60$ ) for all breeds. In the study of Soyeurt et al. (2011), the predictability of calibration equations was also evaluated using, for example, the coefficients of determination, which ranged from 0.68 up to 1 for the FA evaluated in our study. Other studies that evaluated the use of calibration equations to predict FA composition from MIRS profiles, which are not directly linked to the equations used in our study, also showed that MIRS can be a useful method for predicting FA composition for breeding purposes (Rutten et al., 2009; De Marchi et al., 2011). To obtain accurate estimates of differences between groups, predicted individual FA composition does not have to be $100 \%$ accurate. Because the objective of our study was to compare FA composition of different breeds, which involves estimation of average effects for large groups of animals, the results of the validation studies mentioned above suggest that using predicted FA composition is appropriate for the range of FA presented here. For instance, the content of the FA group total C18:2 is predicted to be smaller than the content of the 2 individual C18:2 FA together, which is most likely due to the low accuracy of the predictions for the FA group total C18:2.

\section{Estimates of Breed Effects, Heterosis, and Recombination}

Breed effects were estimated using a data set from commercial herds including purebred and crossbred cows. Because only a very limited number of herds in- 
Table 6. Estimates of the heterosis and recombination effects

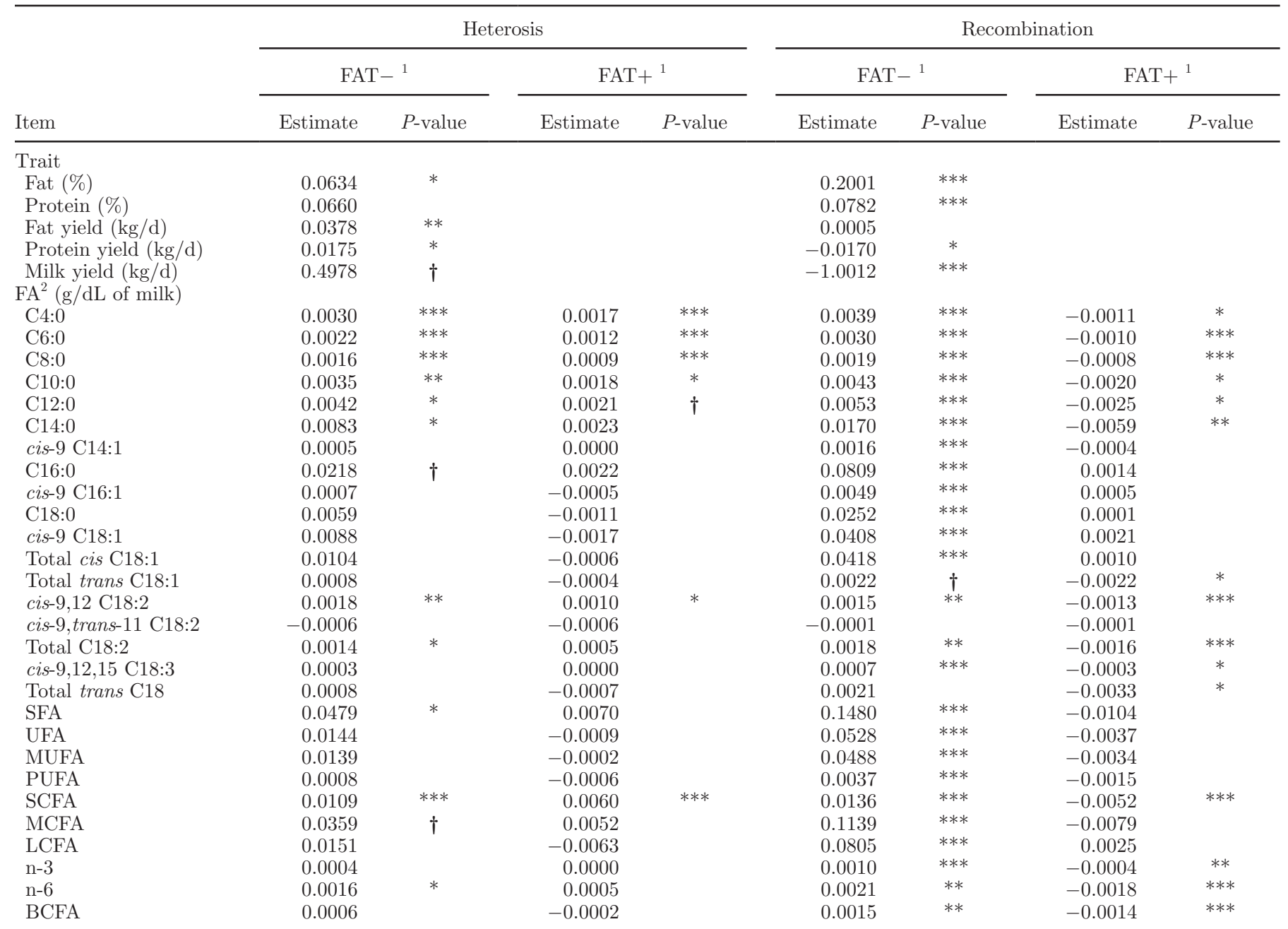

${ }^{1}$ Using the FAT - model, means were predicted regardless of the level of total fat percentage, and using the FAT + model, means were predicted including a fixed regression coefficient of the total fat percentage in milk within the breeds Holstein-Friesian, Meuse-Rhine-Yssel, Dutch Friesian, Groningen White Headed, and Jersey.

${ }^{2} \mathrm{UFA}=$ unsaturated FA; SCFA = short-chain FA; MCFA = medium-chain FA; LCFA = long-chain FA; BCFA = branched-chain FA. $\dagger P<0.10 ;{ }^{*} P<0.05 ;{ }^{* *} P<0.01 ;{ }^{* * *} P<0.001$.

cluded purebred cows of multiple breeds, data of crossbreds was needed to disentangle breed and herd effects. Table 2 shows the number of cows and herds. All herds apart from 2 (1 MRY and 1 DF herd) had crossbred cows. Thus far, a comparison between breeds based on data across a range of commercial herds with different combinations of breeds was only published by Soyeurt et al. (2006b), which included 7 herds with a total of 275 purebred and crossbred cows belonging to 6 different breeds in the Walloon region of Belgium. Betweenbreed differences were estimated on milk basis, giving results expressed as content in milk ( $\mathrm{g}$ of fat/dL of milk). Using the FAT - model, the average individual FA contents for each breed were estimated. As individual FA contents are correlated with fat percentage, individual FA contents were also estimated for each breed, accounting for differences in fat percentage using the FAT+ model (i.e., in Tables 3-5, for the FAT+ model, predicted means at an average fat percentage of 4.7 are shown). As all predictions were performed for FA contents, it may have been expected that individual milk production affected the predictions, due to diluting the FA content. A well-known measure of cow milk production is fat- and protein-corrected milk (FPCM), which is calculated using fat percentage, protein percentage, and milk yield. Breed-specific correlations between FPCM and UFA and SFA based on the unadjusted animal FA profiles ranged from -0.13 to -0.40 , however, whereas correlations between fat percentage and UFA and SFA ranged from 0.54 to 0.96. When analyzing FA expressed as content in milk, fat percentage generally had a significant effect on FA 
content of individual FA and groups of FA. Given the difference in correlation with FA content, FPCM is expected to have a much smaller effect on FA content than fat percentage.

In our study, highly significant effects of heterosis were found for several SCFA. These effects were estimated to be highest for the model without adjusting for differences in fat content. Positive effects of heterosis for fat percentage and fat yield in our study were in line with results found by Ahlborn-Breier and Hohenboken (1991). Recombination is generally expected to have a negative effect, but positive estimates have been reported for fat percentage (Van Der Werf and De Boer, 1989). We found the same result for fat percentage and all FA in the FAT- model, which was expected because FA content is generally positively related with fat percentage. Our results of the FAT + model seem to indicate that when correcting for fat percentage, recombination effects are negative, following the general expectation.

\section{FA Generated Through De Novo Synthesis (SCFA, C12:0, and C14:0)}

The SCFA, C12:0, and C14:0 arise in milk mainly from de novo synthesis within the mammary epithelial cells (Bauman and Griinari, 2003). A considerable part of the variation in production of these FA is, therefore, expected to have a genetic origin. Among the breeds HF, MRY, and DF, no significant differences in the detailed FA composition were found in our study using the FAT + model. These results are in agreement with Maurice-Van Eijndhoven et al. (2011), who reported no significant differences for SCFA, C12:0, and C14:0 between MRY and DF in the Netherlands and Soyeurt et al. (2006b), who reported no significant differences for C12:0 and C14:0 between HF and MRY participating in the Walloon milk recording in Belgium. In our study, JER produced higher contents of SCFA and C14:0 than the other breeds. This was also found by Beaulieu and Palmquist (1995) for C4:0 to C14:0, by White et al. (2001) for C6:0 to C14:0, and by Palladino et al. (2010) for C4:0 to C12:0, although the differences found in the latter study were not significant. Soyeurt et al. (2006b) reported significantly higher contents (g/dL of milk) of C12:0 and C14:0 for JER compared with HF, but differences were not significant when expressed as proportion of total fat. Our results for SCFA and C14:0 for JER were not in line with results reported by Maurice-Van Eijndhoven et al. (2011), who found lower proportions for JER compared with DF and MRY. This might be due to confounding of herd and breed effects in the study by Maurice-Van Eijndhoven et al. (2011). Higher contents of SCFA and C14:0 in JER suggest that this breed has a relatively higher production of FA through de novo synthesis.

\section{C16:0}

The FA C16:0 is one of the major SFA in bovine milk. The FA C16:0 arises in milk through either de novo synthesis or uptake from blood circulation (Bauman and Griinari, 2003). As SFA such as C16:0 are associated with a higher risk of coronary artery disease (e.g., Mensink et al., 2003), possibilities to alter the content of C16:0 in milk is of interest to the dairy industry. In general, our results showed higher contents of C16:0 for JER and lower contents for GWH. The results for C16:0 in our study are in line with results found by MauriceVan Eijndhoven et al. (2011). Similar results for C16:0 in JER compared with HF were reported by Soyeurt et al. (2006b) and Palladino et al. (2010). Contrasting results were only reported by Beaulieu and Palmquist (1995) who reported a higher proportion C16:0 for HF and no difference was found by White et al. (2001).

\section{LCFA}

Fatty acids with more than 16 carbons are obtained by uptake from circulation, which implies that the cow diet has a large influence on the secretion of these FA in milk. Within the cow, however, 2 important systems affect the final milk fat composition: rumen biohydrogenation and $\Delta^{9}$-desaturase activity (Neville and Picciano, 1997). Two essential FA in the human diet that arise in bovine milk directly from the cow diet are cis-9,12,15 C18:3 and cis-9,12 C18:2, together comprising the main fraction of the groups n-3 and n- 6 , respectively. Although these essential FA are present in milk in very low amounts ( $<2 \%$ of total fat), some significant differences were found between breeds. Jerseys produced a higher content of the C18:3 compared with HF, MRY, and DF and a lower content of cis- 9,12 C18:2 compared with all other breeds when adjusting for differences in fat content. The lower production of cis-9,12 C18:2 for JER compared with HF, when analyzed as grams per $100 \mathrm{~g}$ of fat, was also reported by Palladino et al. (2010). For the FA cis-9,12,15 C18:3, contrasting results were found in several other studies (Drackley et al., 2001; Palladino et al. 2010). No differences for these FA were found by Beaulieu and Palmquist (1995) and White et al. (2001).

The FA C18:0 is produced mainly by biohydrogenation in the rumen of PUFA from dietary fat. The trans C18 FA are intermediate products in the biohydrogenation process of C18 PUFA in the rumen and, thus, a result of incomplete biohydrogenation (Bauman and Griinari, 2003). When accounting for differences in fat 
content, the content of C18:0 was significantly higher for GWH compared with JER; however, contrasting results were found without accounting for differences in fat content, showing higher contents for JER and lower for GWH. Soyeurt et al. (2006b) also found a higher C18:0 content for JER compared with HF. Other studies that evaluated proportions of the total fat, however, reported no significant differences between JER and other breeds such as HF and GWH (Beaulieu and Palmquist, 1995; White et al., 2001; Palladino et al., 2010; Maurice-Van Eijndhoven et al., 2011). Evaluating the total trans C18 FA in our study, only using the model without regression on fat percentage, a significantly higher content was found for JER compared with HR and MRY. Thus, the higher content of most LCFA produced by JER is directly related to its higher fat percentage in milk.

The conversion of trans-11 C18:1 into cis-9,trans-11 $\mathrm{C} 18: 2$ via $\Delta^{9}$-desaturase in the udder is the major source of cis-9,trans-11 C18:2 in milk. Although GWH seems to produce a higher content of cis-9,trans-11 C18:2, based on the unadjusted data in the current study, no significant difference among breeds was found following the FAT+ model and even a significant lower mean was predicted for the FAT- model compared with HF and DF. Maurice-Van Eijndhoven et al. (2011) reported a higher proportion of cis-9,trans-11 C18:2 for GWH and a lower proportion for JER. The predicted means of cis-9,trans-11 C18:2 for JER in current study were significantly higher compared with HF and MRY when evaluating the results of the FAT+ model. Contrasting results were found by White et al. (2001) who reported a lower proportion of cis-9,trans-11 C18:2 for JER compared with HF. $\Delta^{9}$-Desaturase plays an important role in determining the total proportion of UFA in milk by desaturation of FA, resulting in, for example, cis-9,trans-11 C18:2, C14:1, C16:1, and C18:1 with a double bond at cis-9. Especially these UFA with a cis double bound are reported as having an altering effect on the risk of development of coronary artery disease (Mensink et al., 2003). In our study, the results of the FAT + model for JER showed significantly higher content of cis-9 C14:1 and lower content of cis-9 C16:1 and cis-9 C18:1. In agreement, White et al. (2001) reported lower proportions cis-9 C16:1 and cis-9 C18:1 for JER compared with HF. For cis-9 C18:1, both, Beaulieu and Palmquist (1995) and Palladino et al. (2010) also found lower proportions for JER compared with HF. The differences between the models reveal that the higher content of cis-9 16:1 and cis-9 C18:1 produced by JER is also directly related to the higher fat percentage in milk of JER.

\section{Major Genes Affecting FA Composition}

Despite potential differences between cow diets in our and other studies, our results were generally in agreement with breed differences reported in other studies. This confirms that differences in FA are partly determined by genetics. Several studies reported that polymorphisms in the genes encoding the acyl CoA:diacylglycerol acyltransferase 1 (DGAT1) and stearoyl-CoA desaturase 1 (SCD1) enzymes explain an important part of the variation in milk fat composition (e.g., Grisart et al., 2002; Schennink et al., 2007; Schennink et al., 2008). Potential differences in allele frequencies between breeds for these genes might explain part of the observed breed differences. For some Jersey and several Holstein populations, the frequencies of the DGAT1 232K and SCD1 293A alleles have been reported in the literature. The frequency of the DGAT1 $232 \mathrm{~K}$ allele in Holsteins was reported as ranging from 0.27 to 0.60 (Spelman et al., 2002; Thaller et al., 2003; Kaupe et al., 2004; Lacorte et al., 2006; Schennink et al., 2007) and somewhat higher in JER, ranging from 0.69 to 0.88 (Spelman et al., 2002; Kaupe et al., 2004). For example, Grisart et al. (2002) and Winter et al. (2002) reported an association of the DGAT1 232K allele with a higher fat percentage in milk and Schennink et al. (2007) reported an association with a higher proportion C16:0. Thus, these reported differences in frequencies for the DGAT1 $232 \mathrm{~K}$ allele in HF and JER, and the reported effects of this allele, are in line with the results of our study.

The frequency of the SCD1 293A allele in Holsteins was reported as ranging from 0.56 to 0.73 (Kgwatalala et al., 2007; Mele et al., 2007; Macciotta et al., 2008; Milanesi et al., 2008; Schennink et al., 2008), whereas it was reported to be higher in JER, ranging from 0.94 to 0.95 (Kgwatalala et al., 2007; Moioli et al., 2007). The SCD1 293A allele was reported to be associated with a higher proportion of $\mathrm{C} 14: 0$, cis-9 $\mathrm{C} 14: 1$, and C18:0 and a lower proportion of cis-9 C16:1 (Schennink et al., 2008), which is in line with the differences found between JER and HF in our study, except for C18:0.

The above-described differences in allele frequencies and the associations of the alleles with the FA composition indicate that the differences between $\mathrm{HF}$ and JER, to a large extent, may be the result of differences in allele frequencies at the DGAT1 and $S C D 1$ genes. Whether or not, for instance, differences between JER and GWH in our study can also be attributed to differences in allele frequencies at those genes is currently unknown, as the allele frequencies for GWH, as well as for DF and MRY, are currently not known. 


\section{CONCLUSIONS}

Breed differences were found in the detailed FA composition and content of individual FA. Especially for FA content in milk produced through de novo synthesis (SCFA, C12:0, C14:0, and partly C16:0), differences were found for JER and GWH compared with the breeds HF, MRY, and DF. For FA having more than 16 carbons, breed differences in content in milk were found for FA that arise in milk directly from the cow diet (cis-9,12 C18:2 and cis-9,12,15 C18:3) of which the total amount is influenced by biohydrogenation in the rumen. Breed differences were also found for FA content in milk in which $\Delta^{9}$-desaturase plays a role $($ cis9 C14:1, cis-9 C16:1, and cis-9 C18:1). No significant differences were found between the predicted means of any individual FA or group of FA among the breeds HF, MRY, and DF. Comparing predicted means from both models, including and excluding a regression on fat percentage, clearly indicated that the detailed FA composition in content in milk, especially SCFA and MCFA, is related to the total fat percentage in all studied breeds.

\section{ACKNOWLEDGMENTS}

This study was financially supported by the Ministry of Economic Affairs, Agriculture and Innovation (The Hague, the Netherlands; Program "Kennisbasis Research," code: KB-04-002-021 and KB-05-003-041). CRV BV (Arnhem, the Netherlands) is thanked for the contribution of the data including mid-infrared profiles. The RobustMilk project team is thanked for the use of the calibration equations. The RobustMilk project is financially supported by the European Commission under the Seventh Research Framework Program Grant Agreement KBBE-211708. This publication represents the views of the authors, not the European Commission, and the Commission is not liable for any use that may be made of the information. Furthermore, Johan van Arendonk (Wageningen University, Animal Breeding and Genomics Centre, Wageningen, the Netherlands) as well as Mari Smits and Roel Veerkamp (Wageningen UR Livestock Research, Animal Breeding and Genomics Centre, Lelystad, the Netherlands) and Sipke Joost Hiemstra [Centre for Genetic Resources, the Netherlands (CGN) of Wageningen UR] are thanked for comments on the setup and data analysis of this study.

\section{REFERENCES}

Ahlborn-Breier, G., and W. D. Hohenboken. 1991. Additive and nonadditive genetic effects on milk production in dairy cattle: Evidence for major individual heterosis. J. Dairy Sci. 74:592-602.
Bauman, D. E., and J. M. Griinari. 2003. Nutritional regulation of milk fat synthesis. Annu. Rev. Nutr. 23:203-227.

Baumgard, L. H., J. K. Sangster, and D. E. Bauman. 2001. Milk fat synthesis in dairy cows is progressively reduced by increasing supplemental amounts of trans-10, cis-12 conjugated linoleic acid (CLA). J. Nutr. 131:1764-1769.

Beaulieu, A. D., and D. L. Palmquist. 1995. Differential effects of high fat diets on fatty acid composition in milk of Jersey and Holstein cows. J. Dairy Sci. 78:1336-1344.

De Marchi, M., M. Penasa, A. Cecchinato, M. Mele, P. Secchiari, and G. Bittante. 2011. Effectiveness of mid-infrared spectroscopy to predict fatty acid composition of Brown Swiss bovine milk. Animal 5:1653-1658.

DePeters, E. J., J. F. Medrano, and B. A. Reed. 1995. Fatty acid composition of milk fat from three breeds of dairy cattle. Can. J. Anim. Sci. 75:267-269.

Dijkstra, J., S. M. van Zijderveld, J. A. Apajalahti, A. Bannink, W. J. J. Gerrits, J. R. Newbold, H. B. Perdok, and H. Berends. 2011. Relationships between methane production and milk fatty acid profiles in dairy cattle. Anim. Feed Sci. Technol. 166-67:590-595.

Drackley, J. K., A. D. Beaulieu, and J. P. Elliott. 2001. Responses of milk fat composition to dietary fat or nonstructural carbohydrates in Holstein and Jersey cows. J. Dairy Sci. 84:1231-1237.

Gilmour, A. R., B. J. Gogel, B. R. Cullis, and R. Thompson. 2009. ASReml User Guide Release 3.0. VSN International Ltd., Hemel Hempstead, UK.

Grisart, B., W. Coppieters, F. Farnir, L. Karim, C. Ford, P. Berzi, N. Cambisano, M. Mni, S. Reid, P. Simon, R. Spelman, M. Georges, and R. Snell. 2002. Positional candidate cloning of a QTL in dairy cattle: Identification of a missense mutation in the bovine DGAT1 gene with major effect on milk yield and composition. Genome Res. 12:222-231.

Kaupe, B., A. Winter, R. Fries, and G. Erhardt. 2004. DGAT1 polymorphism in Bos indicus and Bos taurus cattle breeds. J. Dairy Res. 71:182-187.

Kgwatalala, P. M., E. M. Ibeagha-Awemu, J. F. Hayes, and X. Zhao. 2007. Single nucleotide polymorphisms in the open reading frame of the stearoyl-CoA desaturase gene and resulting genetic variants in Canadian Holstein and Jersey cows. DNA Seq. 18:357-362.

Lacorte, G. A., M. A. Machado, M. L. Martinez, A. L. Campos, R. P. Maciel, R. S. Verneque, R. L. Teodoro, M. G. C. D. Peixoto, M. R. S. Carvalho, and C. G. Fonseca. 2006. DGAT1 K232A polymorphism in Brazilian cattle breeds. Genet. Mol. Res. 5:475-482.

Lawless, F., C. Stanton, P. L'Escop, R. Devery, P. Dillon, and J. J. Murphy. 1999. Influence of breed on bovine milk cis-9, trans11-conjugated linoleic acid content. Livest. Prod. Sci. 62:43-49.

Macciotta, N. P. P., M. Mele, G. Conte, A. Serra, M. Cassandro, R. Dal Zotto, A. C. Borlino, G. Pagnacco, and P. Secchiari. 2008. Association between a polymorphism at the stearoyl CoA desaturase locus and milk production traits in Italian Holsteins. J. Dairy Sci. 91:3184-3189.

Maurice-Van Eijndhoven, M. H. T., S. J. Hiemstra, and M. P. L. Calus. 2011. Short communication: Milk fat composition of 4 cattle breeds in the Netherlands. J. Dairy Sci. 94:1021-1025.

Maurice-Van Eijndhoven, M. H. T., H. Soyeurt, F. Dehareng, and M. P. L. Calus. 2013. Validation of fatty acid predictions in milk using mid-infrared spectrometry across cattle breeds. Animal 7:348354.http://dx.doi.org/10.1017/S1751731112001218

Mele, M., G. Conte, B. Castiglioni, S. Chessa, N. P. P. Macciotta, A. Serra, A. Buccioni, G. Pagnacco, and P. Secchiari. 2007. Stearoylcoenzyme A desaturase gene polymorphism and milk fatty acid composition in Italian Holsteins. J. Dairy Sci. 90:4458-4465.

Mele, M., R. Dal Zotto, M. Cassandro, G. Conte, A. Serra, A. Buccioni, G. Bittante, and P. Secchiari. 2009. Genetic parameters for conjugated linoleic acid, selected milk fatty acids, and milk fatty acid unsaturation of Italian Holstein-Friesian cows. J. Dairy Sci. 92:392-400.

Mensink, R. P., P. L. Zock, A. D. M. Kester, and M. B. Katan. 2003. Effects of dietary fatty acids and carbohydrates on the ratio of serum total to HDL cholesterol and on serum lipids and apoli- 
poproteins: A meta-analysis of 60 controlled trials. Am. J. Clin. Nutr. 77:1146-1155.

Milanesi, E., L. Nicoloso, and P. Crepaldi. 2008. Stearoyl CoA desaturase (SCD) gene polymorphisms in Italian cattle breeds. J. Anim. Breed. Genet. 125:63-67.

Moioli, B., G. Contarini, A. Avalli, G. Catillo, L. Orrù, G. De Matteis, G. Masoero, and F. Napolitano. 2007. Short communication: Effect of stearoyl-coenzyme A desaturase polymorphism on fatty acid composition of milk. J. Dairy Sci. 90:3553-3558.

Neville, M. C., and M. F. Picciano. 1997. Regulation of milk lipid secretion and composition. Annu. Rev. Nutr. 17:159-183.

Palladino, R. A., F. Buckley, R. Prendiville, J. J. Murphy, J. Callan, and D. A. Kenny. 2010. A comparison between Holstein-Friesian and Jersey dairy cows and their $\mathrm{F}_{1}$ hybrid on milk fatty acid composition under grazing conditions. J. Dairy Sci. 93:2176-2184.

Palmquist, D. L., K. Stelwagen, and P. H. Robinson. 2006. Modifying milk composition to increase use of dairy products in healthy diets. Anim. Feed Sci. Technol. 131:149-153.

Rutten, M. J. M., H. Bovenhuis, K. A. Hettinga, H. J. F. van Valenberg, and J. A. M. van Arendonk. 2009. Predicting bovine milk fat composition using infrared spectroscopy based on milk samples collected in winter and summer. J. Dairy Sci. 92:6202-6209.

Schennink, A., J. M. L. Heck, H. Bovenhuis, M. H. P. W. Visker, H J. F. van Valenberg, and J. A. M. van Arendonk. 2008. Milk fatty acid unsaturation: Genetic parameters and effects of stearoyl-CoA desaturase $(S C D 1)$ and acyl CoA:diacylglycerol acyltransferase 1 (DGAT1). J. Dairy Sci. 91:2135-2143.

Schennink, A., W. M. Stoop, M. H. P. W. Visker, J. M. L. Heck, H. Bovenhuis, J. J. van der Poel, H. J. F. van Valenberg, and J. A. M. van Arendonk. 2007. DGAT1 underlies large genetic variation in milk-fat composition of dairy cows. Anim. Genet. 38:467-473.

Smet, K., J. De Block, S. De Campeneere, D. De Brabander, L. Herman, K. Raes, K. Dewettinck, and K. Coudijzer. 2009. Oxidative stability of UHT milk as influenced by fatty acid composition and packaging. Int. Dairy J. 19:372-379.

Soyeurt, H., P. Dardenne, F. Dehareng, G. Lognay, D. Veselko, M. Marlier, C. Bertozzi, P. Mayeres, and N. Gengler. 2006a. Estimating fatty acid content in cow milk using mid-infrared spectrometry. J. Dairy Sci. 89:3690-3695.

Soyeurt, H., P. Dardenne, A. Gillon, C. Croquet, S. Vanderick, P. Mayeres, C. Bertozzi, and N. Gengler. 2006b. Variation in fatty acid contents of milk and milk fat within and across breeds. J. Dairy Sci. 89:4858-4865.
Soyeurt, H., F. Dehareng, N. Gengler, S. McParland, E. Wall, D. P. Berry, M. Coffey, and P. Dardenne. 2011. Mid-infrared prediction of bovine milk fatty acids across multiple breeds, production systems, and countries. J. Dairy Sci. 94:1657-1667.

Soyeurt, H., A. Gillon, S. Vanderick, P. Mayeres, C. Bertozzi, and N. Gengler. 2007a. Estimation of heritability and genetic correlations for the major fatty acids in bovine milk. J. Dairy Sci. 90:4435-4442.

Soyeurt, H., S. Tsuruta, I. Misztal, and N. Gengler. 2007b. Principal components approach for estimating heritability of mid-infrared spectrum in bovine milk. J. Dairy Sci. 90(Suppl. 1):596-597. (Abstr.)

Spelman, R. J., C. A. Ford, P. McElhinney, G. C. Gregory, and R. G. Snell. 2002. Characterization of the DGAT1 gene in the New Zealand dairy population. J. Dairy Sci. 85:3514-3517.

Sterk, A., B. E. O. Johansson, H. Z. H. Taweel, M. Murphy, A. M. van Vuuren, W. H. Hendriks, and J. Dijkstra. 2011. Effects of forage type, forage to concentrate ratio, and crushed linseed supplementation on milk fatty acid profile in lactating dairy cows. J. Dairy Sci. 94:6078-6091.

Stoop, W. M., J. A. M. van Arendonk, J. M. L. Heck, H. J. F. van Valenberg, and H. Bovenhuis. 2008. Genetic parameters for major milk fatty acids and milk production traits of Dutch HolsteinFriesians. J. Dairy Sci. 91:385-394.

Thaller, G., W. Kramer, A. Winter, B. Kaupe, G. Erhardt, and R. Fries. 2003. Effects of DGAT1 variants on milk production traits in German cattle breeds. J. Anim. Sci. 81:1911-1918.

Van Der Werf, J. H. J., and W. De Boer. 1989. Estimation of genetic parameters in a crossbred population of black and white dairy cattle. J. Dairy Sci. 72:2615-2623.

White, S. L., J. A. Bertrand, M. R. Wade, S. P. Washburn, J. T. Green, and T. C. Jenkins. 2001. Comparison of fatty acid content of milk from Jersey and Holstein cows consuming pasture or a total mixed ration. J. Dairy Sci. 84:2295-2301.

Wilmink, J. B. M. 1987. Adjustment of test-day milk, fat and protein yield for age, season and days-in-milk. Livest. Prod. Sci. $16: 335-348$

Winter, A., W. Krämer, F. A. O. Werner, S. Kollers, S. Kata, G. Durstewitz, J. Buitkamp, J. E. Womack, G. Thaller, and R. Fries. 2002. Association of a lysine-232/alanine polymorphism in a bovine gene encoding acyl-CoA:diacylglycerol acyltransferase (DGAT1) with variation at a quantitative trait locus for milk fat content. Proc. Natl. Acad. Sci. USA 99:9300-9305. 